\title{
Image Enlargement Based on Proportional Salient Feature
}

\author{
I Komang Somawirata, Keiichi Uchimura, and Gou Koutaki
}

\begin{abstract}
This paper proposes an image enlargement method that produces proportional salient content of image magnification. To obtain the proportional salient image content: first, we enlarge the source image to the high size of the target image using uniform enlarging. Second, we slice the image into sections from top to bottom following the minimum energy and detect the salient feature of the image. Third, we enlarge the slice of the image region that does not containthe salient feature of the image to the full size of the target image. The proposed method has been tested in several images, such as akiyo, butterfly, cameraman, canoe, dolphin, and parrot. The experimental results show that the proposed method results in a proportional content for image enlargement in the different ratios compared with the comparison method.
\end{abstract}

Index Terms - image enlargement, energy, slice, window.

\section{INTRODUCTION}

C urrently, the various sizes and ratios of displays have been presented. The ratios of display size rang from $3: 2,4: 3$, 5:4, 16:9, 16:10 and 17:9 respectively for the width and height. It requires a good image enlargement method.

Image enlargement is chang the smaller image size to a larger image size. In other words, image enlargement produces a high-resolution (HR) [1,2] image. We distinguish the enlargement of an image into two, namely uniform and nonuniform image enlargement. Nonuniform image enlargement produces image magnification in different ratios. There are two problems in image magnification; The first is having an enlarged image look rough, jagged edge area and blurry; the second is non-proportional content, especially in image magnification in different ratios.

Many methods [1-5] have been developed for image enlargement to obtain good image quality. The image enlargement method with edge improvement is also done to result in good image quality $[3,4]$. Image enlargement based on regularity on the geometric is proposed by [3]. This method uses covariance-based adaptive interpolation, especially in

Manuscript received November, 2013. This work was supported by Directorate General of Higher Education Ministry of National Education (DGHE) of Indonesia which provide scholarship.

The first and second authors are with Computer Science and Electrical Engineering, Graduate School of Science and Technology, Kumamoto University, Japan, (e-mail:kmgsomawirata@navi.cs.kumamoto-u.ac.jp; uchimura@cs.kumamoto-u.ac.jp)

The third author is with Priority Organization for Innovation and Excellence, Kumamoto University, Kumamoto 860-855, Japan (koutaki@cs.kumamoto-u.ac.jp). edges and pixels near the edge. They use bilinear interpolation for nonedge pixels. This method is called NEDI. However, NEDI method is only for uniform image enlargement and it is based on two scale factors. The reverse diffusion interpolation (RDI) [4] to recover sharp edges on HR image is capable of reducing highfrequency in the edge image. In this method, reverse diffusion serves as a nonlinear high-pass filter. Partial volume correction with reverse diffusion needs 200 iterations, which causes the RDI method require long computation time. The RDI method is only used to enlarge the image in the same ratios. Image enlargement methods based on the linear weighting technique to produce a better HR image are implemented on weighted sum of linear extrapolations (WLE) [5]. The WLE method uses eight pixel samples to determine one pixel interpolation. The quality of the image enlargement result using the WLE method is slightly higher compared with classic methods such as bilinear interpolation and cubic interpolation.

The methods [1-5] are only for image enlargement in uniform size, and scale factor must be an integer value. They are difficult to implement in different ratios and shapes. We solve these problems by using image enlargement based on window kernel. This method is easily used to enlarge the image in different ratios and shapes.

One of the popular resizing methods is seam carving [6]. Seam carving is a novel content-aware resizing scheme. This method uses gradient magnitude for measuring the image importance. However, visual distortions often occur when the content of image is complex backgrounds. To overcome this problem, many researchers, especially on image retargeting, start to focus on dealing with visual salience while suppressing nonsalient regions [6-11]. These methods resize image to smaller size in different ratios. Nevertheless, resizing an image to a bigger size from the source image produces distortion between the left side and the right side of image carving. This is caused by the interpolation of a pixel in the carving of the image.

In this paper, to overcome the problem, we propose an image enlargement method by combining the scaling method based on the window kernel, the image slicing method, and feature detection, which produces the proportional content of image enlargement.

\section{Proposed Method}

This research proposes a method of image enlargement that combines the scaling methods based on window kernel 


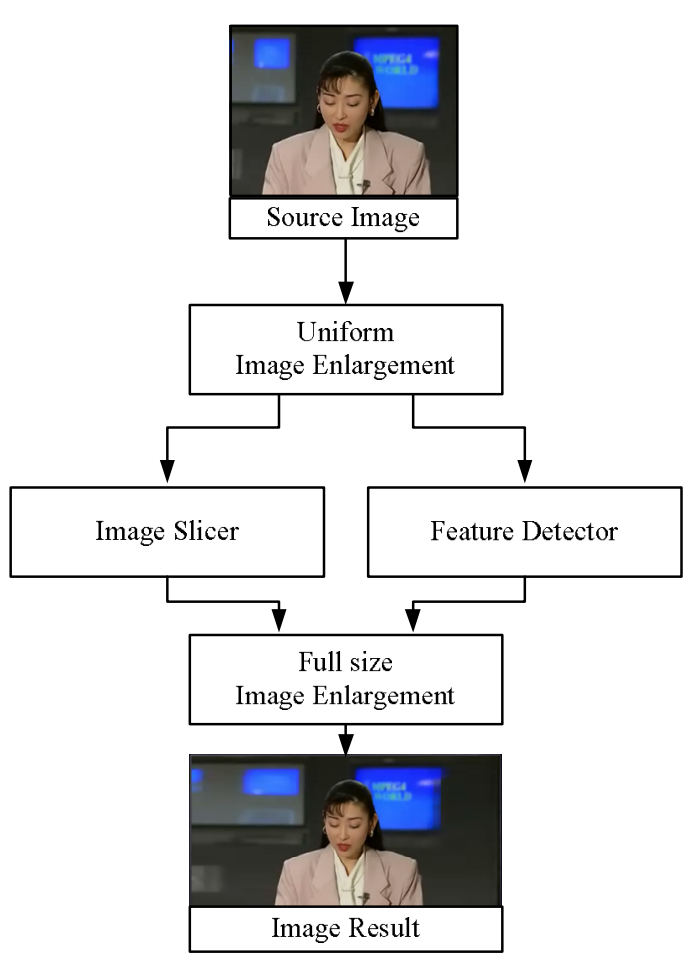

Fig.1. Diagram block of proposed method

image slicing and feature detection methods, as shown in Fig.1. There are four main blocks, namely uniform image enlargement, image slicer, feature detector and full size image enlargement. Uniform image enlargement is used to enlarge the source image uniformly to the high size of the target image, as shown in Fig.4. (b). Image slicer is used to slice an image into several parts. Feature detector is used to detect the important features in the image, whereas image enlargement is used to enlarge the slice image region with different scale factors based on the important features of the image.

\section{A. Image Enlargement Based on the Window Kernel}

The image enlargement using window kernel is illustrated in Fig.2. This method is used to enlarge the source image in the same ratio or in a different ratio. In this case, the method is used to the uniform and full-size images enlarged. The source image is the selected nonsalient feature, that will be enlarged to full-size image, as shown in Fig.2.

The steps of image enlargement based on the window kernel are presented as follows:

- The first step is finding the corresponding coordinates in the source image for the sample pixel kernel, as shown in (1) on Fig.2. The corresponding coordinates $\left(c_{h}\right.$ and $\left.c_{w}\right)$ are obtained by dividing the height $\left(n_{1}\right)$ and width $\left(n_{2}\right)$ coordinates of the target image with the scale factors $\left(S_{h}\right.$ and $S_{w}$ ), as shown in Eq. (1).

$$
c_{h}=\frac{n_{1}}{S_{h}} \quad \text { and } \quad c_{w}=\frac{n_{2}}{S_{v}}
$$

- The second step is taking four pixels for the sample pixel window (ws) as shown in (2) Fig.2 and weight window is calculted based on Fig. 3.

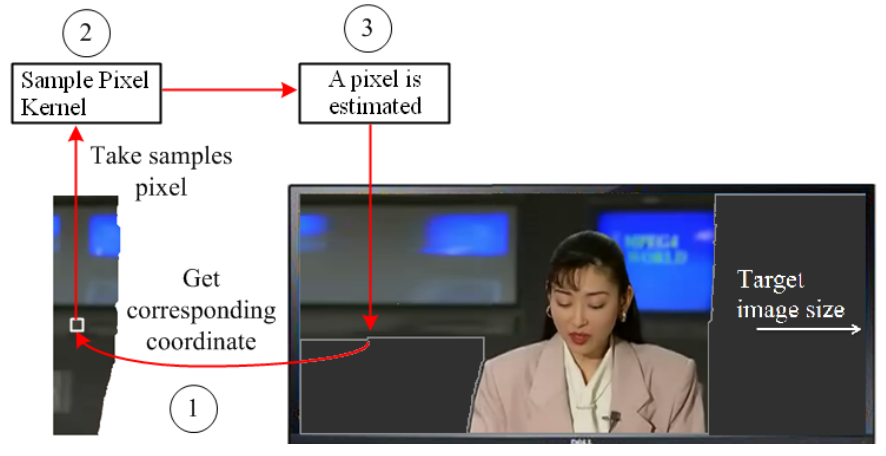

Fig. 2. Image enlargement based on the window kernel

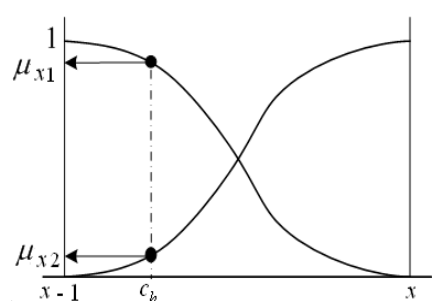

(a)

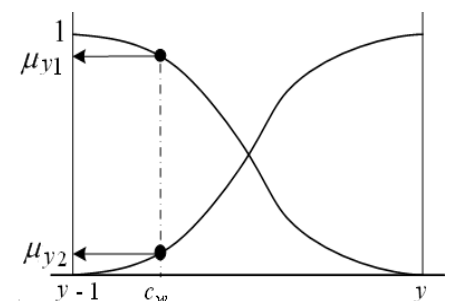

(b)
Fig.3. Curve weighting. (a) and (b) are weight curve for $x$ and $y$ coordinates, respectively

- The third step is calculating one pixel for filling the corresponding coordinate in the target image size.

- Step 1 to 3 are repeated until all the empty coordinates in the target image are filled by pixel.

1) Sample pixel window. The sample pixel window contains four pixels, which is placed in the $2 \times 2$ window size. The fourth sample pixel is obtained from the source image. The corresponding coordinate in the source image $(x$ and $y)$ is obtained by using Eq. (2).

$$
x=\left\lceil c_{h}\right\rceil \text { and } y=\left\lceil c_{w}\right\rceil
$$

Referring to Eq.(1), there are three rules to fill the sample pixel window.

- If the value of $c_{h}$ is a fraction and the value of $c_{w}$ is an integer, then the element of the sample pixel is as in Eq. (3).

$$
w_{s}=\left|\begin{array}{cc}
f(x-1, y) & \frac{f(x-1, y-1)+f(x-1, y)}{2} \\
f(x, y) & \frac{f(x, y-1)+f(x, y)}{2}
\end{array}\right|
$$

- If the value of $c_{h}$ is an integer and the $c_{w}$ is a fraction, then the element of the sample pixel is as in Eq. (4).

$$
w_{s}=\left|\begin{array}{cc}
\frac{f(x-1, y-1)+f(x, y-1)}{2} & \frac{f(x-1, y)+f(x, y)}{2} \\
f(x, y-1) & (x, y)
\end{array}\right|
$$

- If the value of $c_{h}$ is a fraction and the $c_{w}$ is a fraction, then the element of the sample pixel is as in Eq. (5). 


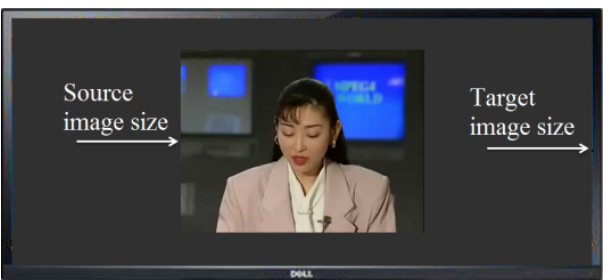

(a)

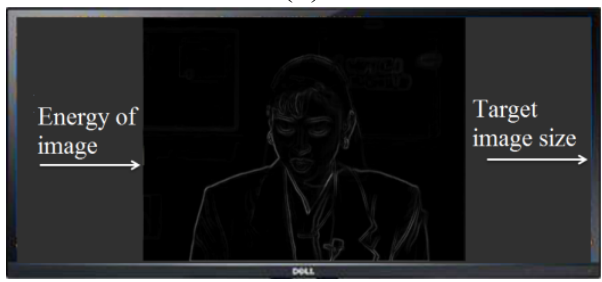

$(d)$

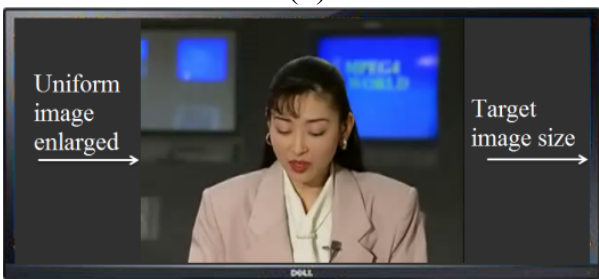

$(g)$

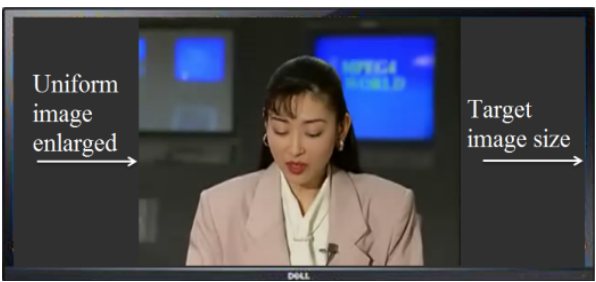

(b)

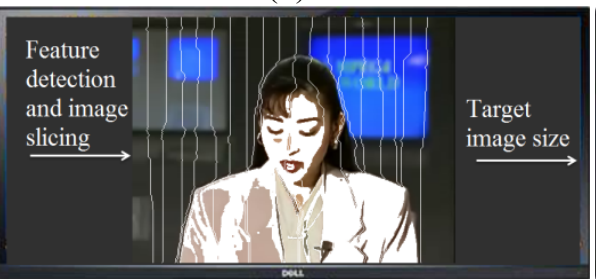

(e)

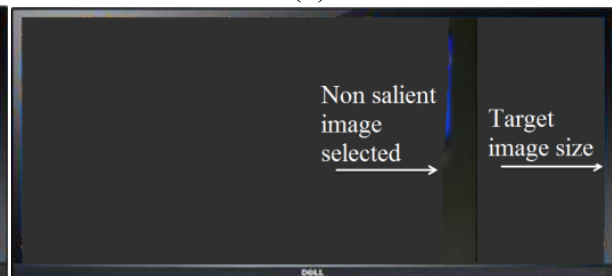

(h)

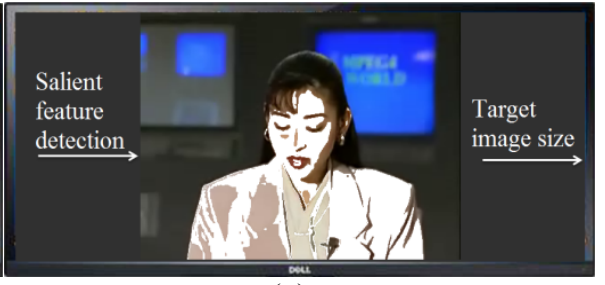

(c)

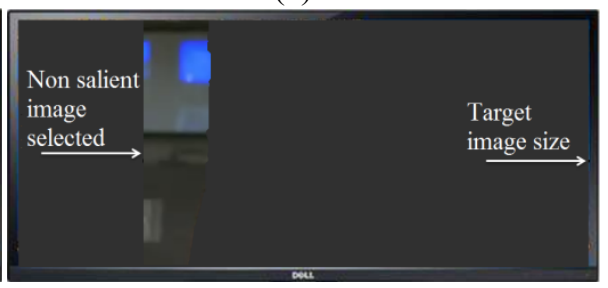

$(f)$

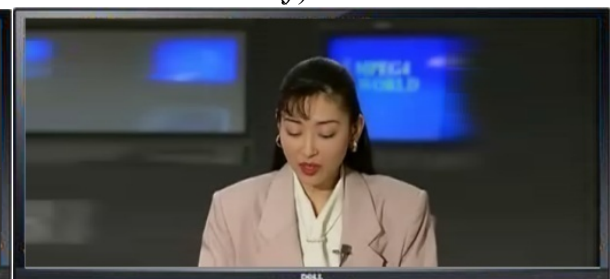

(i)

Fig.4. The step by step result of image enlargement. (a) Source image and target image size, $(b)$ uniform image enlargement, (c) featutre detection, $(d)$ energy of image, $(e)$ image slicing of $(c),(f)$ nonsalient feature slice region selection, $(g)$ slice region selection with fiture content, $(h)$ nonsalient feature slice region selection, $(i)$ image enlargement result.

$$
w_{s}=\left|\begin{array}{cc}
f(x-1, y-1) & f(x-1, y) \\
f(x, y-1) & f(x, y)
\end{array}\right|
$$

Three steps determine the four pixels for window $w_{s}$ that we call by the window kernel.

2) Weight window. We use nonlinear interpolation, which is based on the curve for the image enlargement method as in Fig.3. In the curve weighting method, the shape of curves will determine the enlargement method. If the curve is the linear shape, then it will generate a linear interpolation and vice versa. Both of coordinates $x$ and $y$ on Fig.3. have two weight values. There are $\mu_{x 1}$ and $\mu_{x 2}$ for the $x$ coordinate and $\mu_{y 1}$ and $\mu_{y 2}$ for the $y$ coordinate. The curve weighting on Fig.3. gives weight value greater than the linear weighting value for the pixel nearest the $c_{h}$ and $c_{w}$ points. Equation (6) is used to obtain $\mu_{x 1}$ value for the downhill curve, whereas Eq. (7) is used to obtain the $\mu_{x 2}$ value for the uphill curve.

$$
\begin{gathered}
\mu_{x 1}=1-\frac{1}{1+\left(\frac{c_{h}-x}{0.5}\right)^{2}} \\
\mu_{x 2}=\frac{1}{1+\left(\frac{c_{h}-x}{0.5}\right)^{2}}
\end{gathered}
$$

$\mu_{y 1}$ and $\mu_{y 2}$ are obtained in the same way as $\mu_{x 1}$ and $\mu_{x 2}$; however, the variable $c_{h}$ in Eqs. (6) and (7) is replaced with $c_{w}$. The size of the weight window is $2 \times 2$, which has same size with sample pixel window. The weight window is shown in
Eq. (8).

$$
w_{w}=\left|\begin{array}{ll}
w_{11} & w_{12} \\
w_{21} & w_{22}
\end{array}\right|
$$

The matrix element of $w_{w}$ is obtained by multiplying $\mu_{x \mathrm{i}}$ with $\mu_{y \mathrm{i}}$ as in Eq. (9).

$$
w_{i j}=\mu_{x i} \times \mu_{y j}
$$

wherein, $w_{i j}$ is element matrix of $w_{w}$ with $i=j=1,2$.

3) Filling pixel. A pixel to fill the corresponding coordinate in target image is obtained by multiplying the sample pixel window with weight window.

\section{B. Image Slicer}

Image slicer is used to slice the image into several sections that start at the same distance $\left(L_{w}\right) . L_{w}$ is calculated using Eq. (10).

$$
L_{w}=\frac{w}{n_{s}}
$$

where, $L_{w}$ is the distance between each slice, $w$ is the width of the image, and $n_{s}$ is the number of slices.

Slicing the image starts from top to bottom following the minimum energy of the image. The energy of the image $(E)$ is calculated using Eq. (11).

$$
E=\frac{1}{2}\left(E_{h}+E_{v}\right)
$$

$E$ is two images energy $\left(E_{h}\right.$ and $\left.E_{v}\right)$, in which each point contains the horizontal and vertical absolute derivative approximations; the computations are as in Eq. (12) and Eq.(13) 


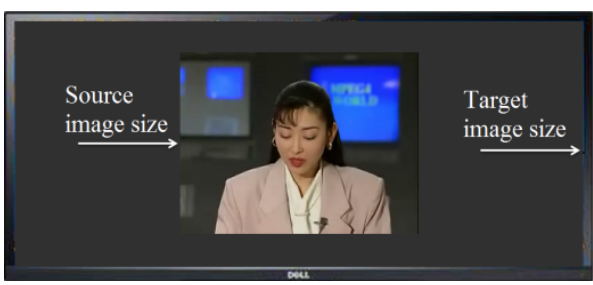

(a) Akiyo

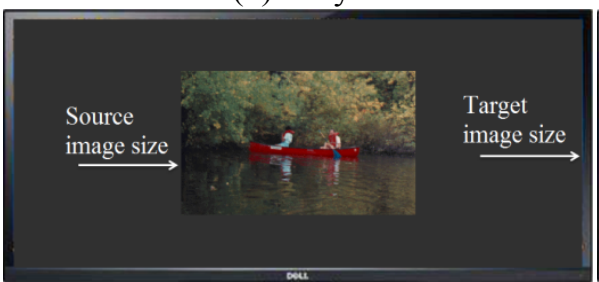

(d) Canoe

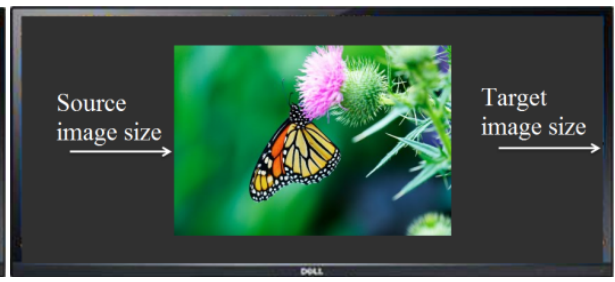

(b) Butterfly

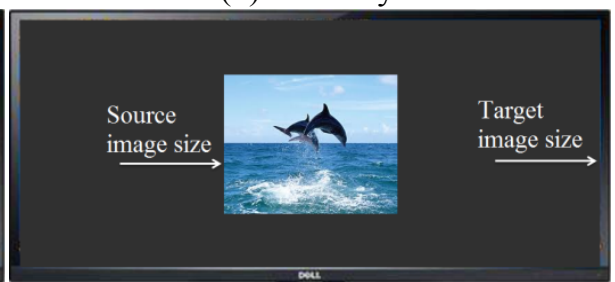

(e) Dolphin

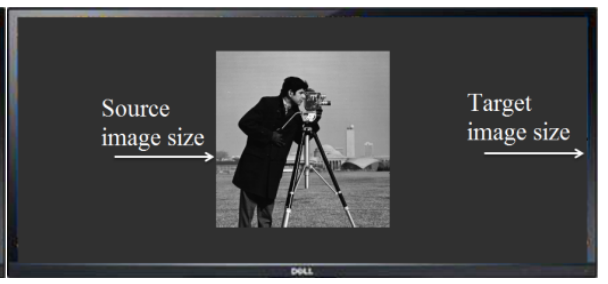

(c) Cameraman

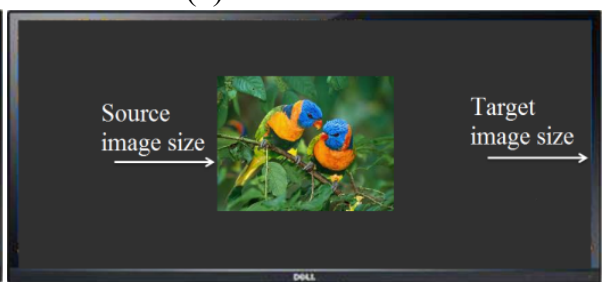

(f) Parrot

Fig.5. Target image size and source images size.

$$
\begin{aligned}
& E_{h}=\left|f * H_{h}\right| \\
& E_{h}=\left|f * H_{h}\right|
\end{aligned}
$$

where, $f$ is the source image. $*$ is convolution. $H_{h}$ and $H_{v}$ are the absolute horizontal and vertical derivative mask with $3 \times 3$ size as in Eq.(14).

$$
H_{h}=\left|\begin{array}{lll}
1 & 0 & -1 \\
2 & 0 & -2 \\
1 & 0 & -1
\end{array}\right| \text { and } H_{v}=\left|\begin{array}{ccc}
1 & 2 & 1 \\
0 & 0 & 0 \\
-1 & -2 & -1
\end{array}\right|
$$

\section{Feature Detector}

Feature detector is to detect salient features of the image. We use a simple formula to obtain the salient feature in the image as shown in Eq. (15).

$$
f_{D}=\left\{\begin{array}{ll}
255 & \text { if } w_{d}
\end{array} \text { э } D_{i}\right.
$$

where, $f_{D}$ is feature detection as shown in Fig. $4(c), w_{d}$ is $3 \times 3$ sample pixel window from image $\hat{f}_{u}, D_{i}$ are sample pixels that consist of several essential elements of the image sample, and $\hat{f}_{u}$ is uniform image enlargement, as shown in Fig.4 $(b)$.

If $D_{i}$ has a part in common with the image $\hat{f}_{u}$, then it will be given a white mark (255) as shown in Fig.4. (c). The white mark indicates the salient feature in the image.

\section{Full size Image Enlargement}

This stage is the final stage of the image enlargement process. Figure $4(e)$ is obtained by combining the image output of image slicer and feature detector. The salient feature on Fig.4. (e) is marked with white. If the part of the slice image contains a salient feature, as in (Fig.4. $(g)$ ), then this section will not be enlarged. However, if the slice image does not contain a salient feature, it will be enlarged to the size of the target image. Figure $4(f)$ and $(h)$ show the selected slice region without white markings as in Fig. $4(e)$. The full size image enlargement is formulated in Eq.(16).

$$
y_{o}= \begin{cases}R_{n}^{l} & \begin{array}{l}
\text { if a slice region of the image } \\
\text { contain salient feature (255) }
\end{array} \\
R_{n}^{S} & \begin{array}{l}
\text { if a slice region of the image does not } \\
\text { containing a salient feature }
\end{array}\end{cases}
$$

where, $y_{o}$ is the full size image enlargement, $R$ is the slice region of image, $n$ is the number of slice region, $S$ is the scale factor and $\uparrow$ is up-scaling by using window kernel method in section $\mathrm{A}$.

\section{EXPERIMENTAL RESULT}

In this experiment, the proposed method is tested for uniform and nonuniform image enlargement. For uniform image enlargement, we use four sample standard images, such as lena, peppers, baboon and the clown. On the other hand, for nonuniform image enlargement, we use six samples such as akiyo, butterfly, cameraman, canoe, dolphin and parrot images as shown in Fig.5. All methods are implemented in Matlab 7 with computer specification $2.9 \mathrm{GHz}$ CPU and 4 GB RAM.

\section{A. Experimental Result}

The proposed method in uniform image enlargement is evaluated using quantitative evaluation, while the nonuniform image enlargement is evaluated using qualitative evaluation.

1) Quantitative evaluation: The image enlargement quality is evaluated using objective quality evaluations such as peak signal-to-noise ratio $(P S N R)$ and mean structural similarity (MSSIM)[12]. We use the default value constant in the SSIM index formula $K=\left[\begin{array}{ll}0.01 & 0.03\end{array}\right]$ (where $K$ is small constant with $K \leq 1$, in this paper, we use $K$ value from experiment in [12]) and $11 \times 11$ Gaussian window size with $\sigma=1.5$.

We create a scenario to test the image enlargement result using the PSNR and MSSIM evaluation as follows: First, we resize the standard image into the smaller size. The sizes of images which would be enlarged were one-half of the standard image size. 


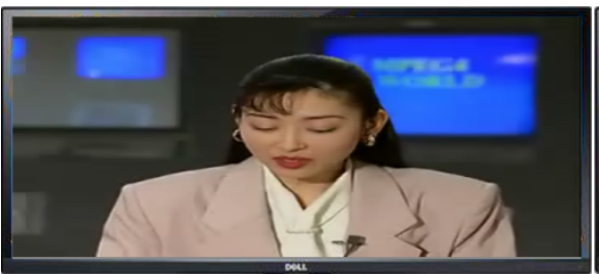

(a) Scale method

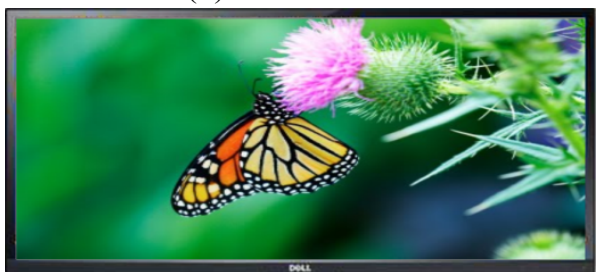

(d) Scale method

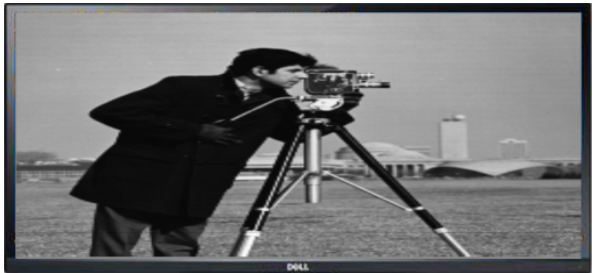

(g) Scale method

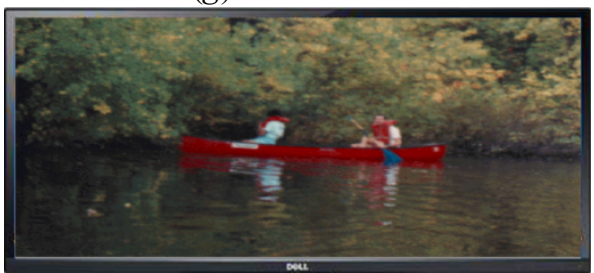

(j) Scale method

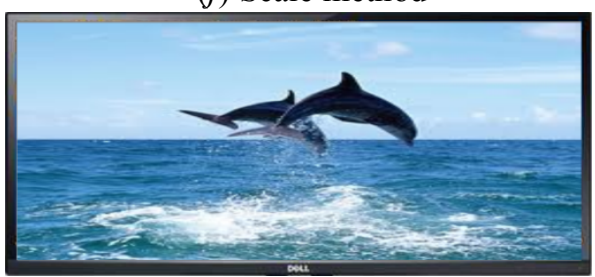

(m) Scale method

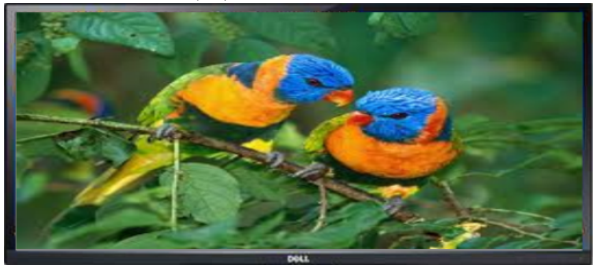

(p) Scale method

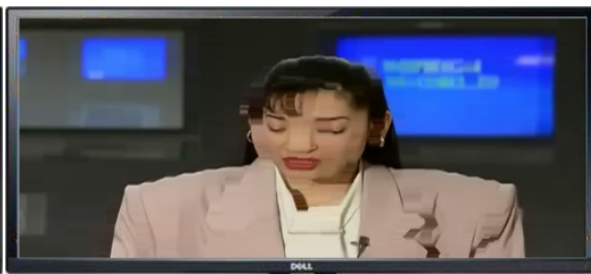

(b) Carving method

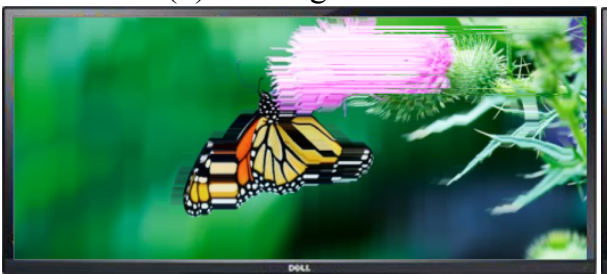

(e) Carving method

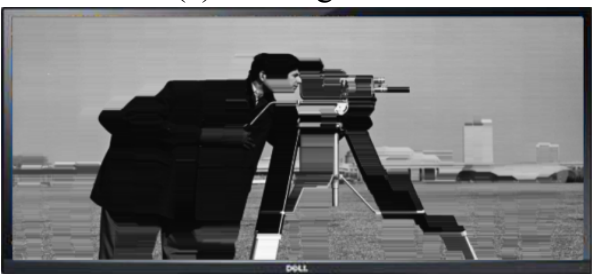

(h) Carving method

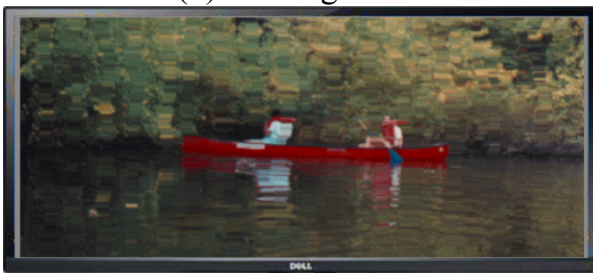

(k) Carving method

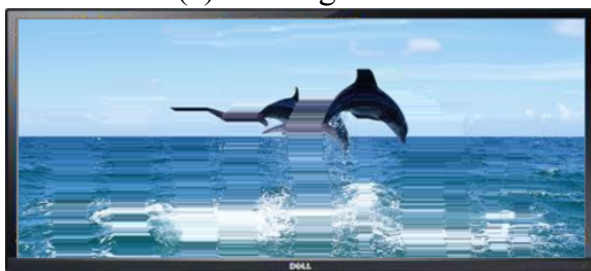

(n) Carving method

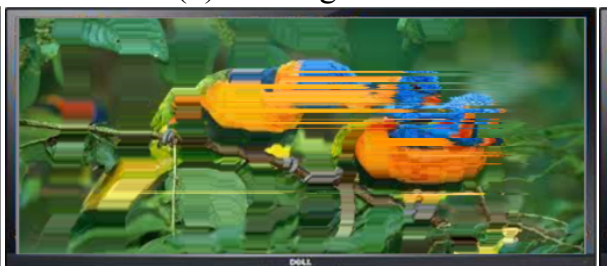

(q) Carving method

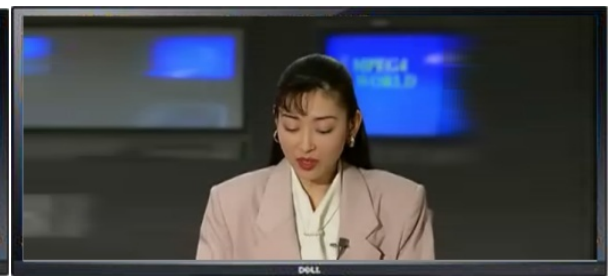

(c) Proposed Method

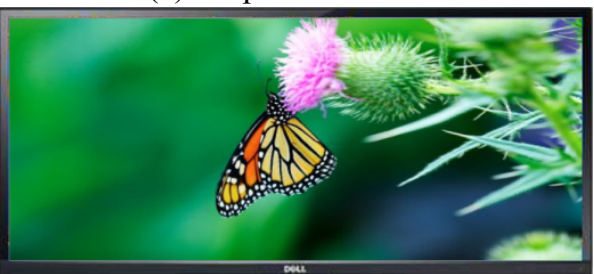

(f) Proposed Method

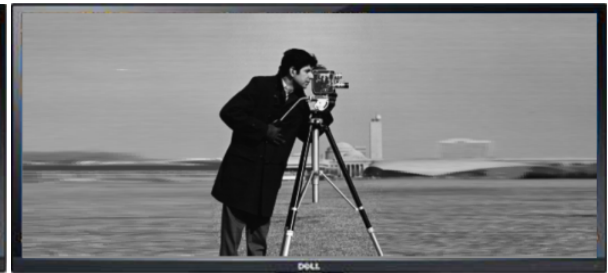

(i) Proposed Method

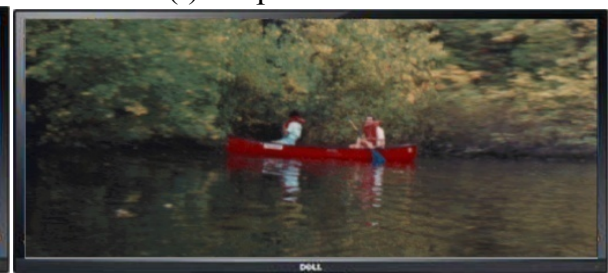

(l) Proposed Method

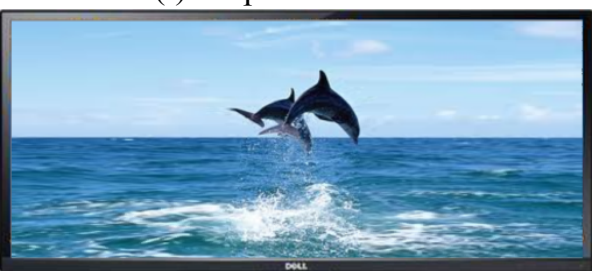

(o) Proposed Method

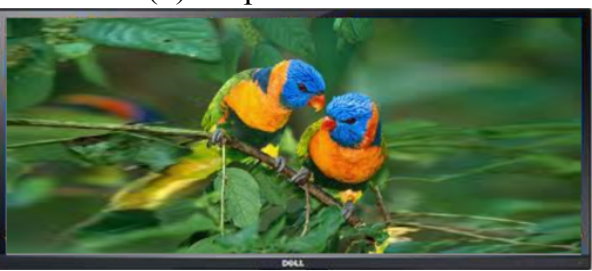

(r) Proposed Method

Fig.6. The comparison of image enlargement result using scale, carving and propoed methods. $(a-c)$ Akiyo image, $(d-f)$ butterfly image, $(g-i)$ cameraman image, $(j-l)$ canoe image, $(m-o)$ dolphin image and $(p-r)$ parrot image.

We use the nearest-neighborhood $(N N)$ method to resize the standard image size. Second, we enlarge the small image size using all methods by the scale factor equal to two to become the standard image size or reference image size.

Figure 7 shows the image quality evaluation using $P S N R$ by scale factor equal to two. The uniform image enlarged using the proposed method has a bigger PSNR value compared with the comparison method. In addition, the image evaluation using MSSIM in Fig.8 shows the proposed method closest to 1, which represents the proposed methods having close similarity to the image reference.

2) Qualitative evaluation: Figure 5 shows the target image size and source image that will be enlarged to the different ratio, whereas, Fig.6. shows the image magnification using scaling techniques, carving and the proposed method.

Image enlargement using scaling and carving methods results in a disproportionate salient content compared with the proposed method. For example, Fig.6 (a) and (b) have an Akiyo that looks fat compared with that in the proposed method. This is the same for the butterfly, cameraman and 

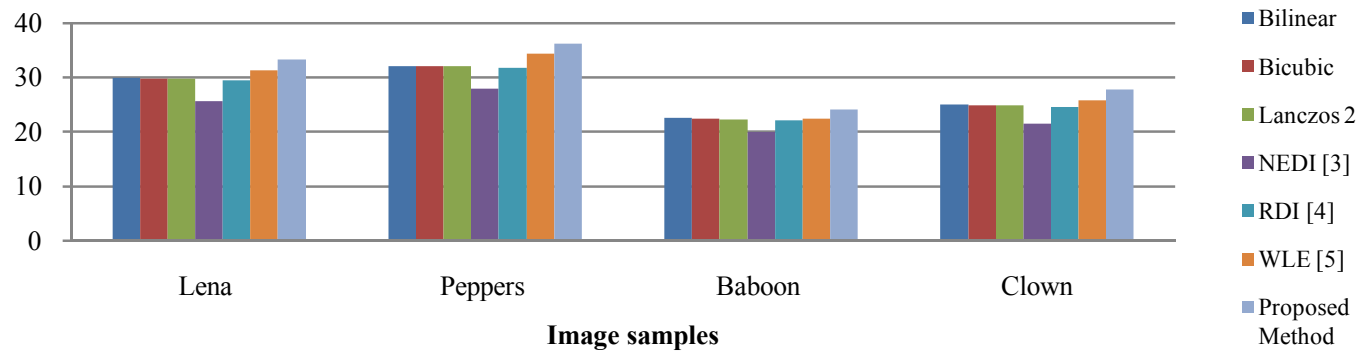

Fig.7. The PSNR comparison of two times image enlargement
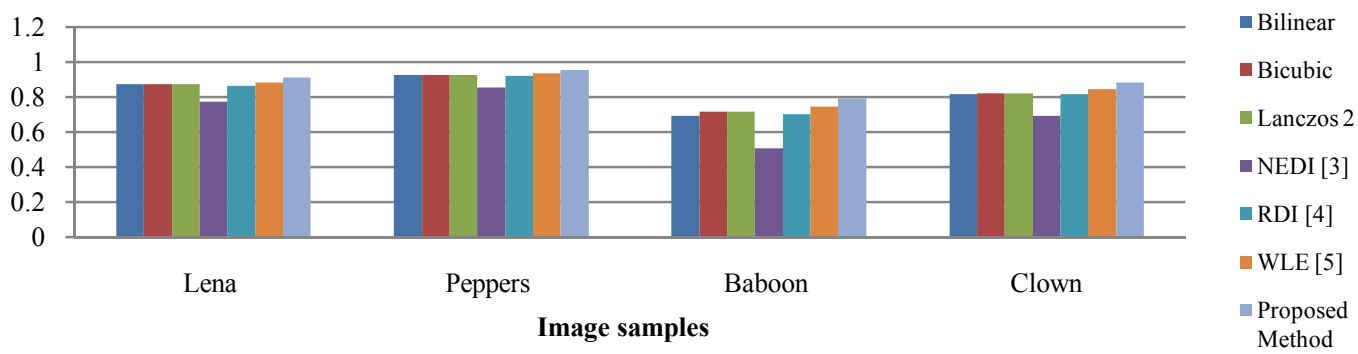

Fig.8. The MSSIM comparison of two times image enlargement

parrot images. On the other hand, the image enlargement of the of canoe and dolphin images using the scaling and the carving method shows more length, as shown in Fig 6. $(j),(k)$, $(m)$ and $(n)$. These are caused by different value of scale factors for height and width of source image. The scale factor for width is greater than that for height.

\section{CONCLUSION}

Proportional salient content image enlargement has been proposed in this paper. This method combines image slices, feature detection and window kernel scaling methods. The proposed method has been evaluated by using PSNR and $M S S I M$, which result in good image quality, and for images enlarged in the different ratio, the proportional salient content is obtained.

For next study, we have plans to implement our method to improve image sequence and feature detection and to obtain an autoselection nonsalient content in an image slice to be enlarged.

\section{REFERENCES}

[1] J. Yang, J.Wright, T. Huang, and Y. Ma, Image Super-Resolution via Sparse Representation, IEEE Trans. on Image Processing, vol 19, pp. $2861-2873,2010$.

[2] W. Dong, L. Zhang, and G. S. X. Wu, Image Deblurring and Superresolution by Adaptive Sparse Domain Selection and Adaptive Regularization, IEEE Trans. on Image Processing, vol 20, pp. 1838 $1857,2011$.

[3] X. Li and M. P. Orchard,New Edge-Directed Interpolation, IEEE Trans. on Image Processing, vol 10, pp. 1521 - 1527, 2001.

[4] O. Salvado, C. M. Hillenbrand and D. L. Wilson, Partial Volume Reduction by Interpolation with Reverse Diffusion, International Journal of Biomedical Imaging, pp. 1 - 13, 2006.

[5] T. Aso, N. Sutake, and T. Yamakau, A Fast and Accurate Image Enlargement Algorithm Employing a Weighted Sum of Linear Extrapolations, Automation Congress, Proceedings. vol. 18, pp. 251 258, 2004.

[6] S. Avidan and A. Shamir, Seam carving for content-aware image resizing, ACM Trans. Graph., vol. 26, no. 3, pp. 267-276, 2007.

[7] N.Dalal, and B.Triggs, Histograms of oriented gradients for human detection, Proc. IEEE Computer Vision and Pattern Recognition (CVPR), vol. 1, pp. 886-893, 2005.
[8] X. Hou, and L. Zhang, Saliency detection, A spectral residual approach, Proc. IEEE Computer Vision and Pattern Recognition (CVPR), pp. 1-8, 2007.

[9] L.Zhang, M.Tong, T.Marks, H.Shan, and G.Cottrell, SUN:A Bayesian framework for saliency using natural statistics, J. Vis., vol. 8, no. 7, pp. $1-20,2008$.

[10] R.Achanta, and S.Susstrunk, Saliency detection for content-aware image resizing, Proc. IEEE Int. Conf. Image Processing (ICIP), pp. 10051008,2009

[11] S. Goferman, L. Z. Manor, and A . Tal, Context-aware saliency detection, Proc. IEEE Computer Vision and Pattern Recognition (CVPR), pp. 2376-2383, 2010.

[12] Z. Wang, A. C. Bovik, H. R. Sheikh, and E. P. Simoncelli, Image quality assessment: From error visibility to structural similarity, IEEE Trans. on Image Processing, vol. 13, no. 4, pp. 600-612, 2004.

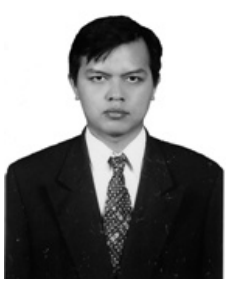

I Komang Somawirata. received bachelor degree in Electrical Engineering from National Institute of Technology Malang Indonesia in 1993-1998, and Master degree in the same major from Brawijaya University, Indonesia in 2004-2007. He is a lecturer in Elektrical Engineering Departmen of National Institute of Technology from 2000 .

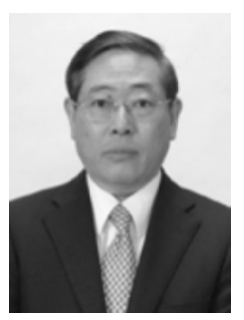

Keiichi Uchimura, received the B.Eng. and M.Eng. degrees from Kumamoto University, Kumamoto, Japan, in 1975 and 1977, respectively, and the Ph.D. degree from Tohoku University, Miyagi, Japan, in 1987. He is currently a Professor in the Graduate School of Science and Technology, Kumamoto University. $\mathrm{He}$ is engaged in research on intelligent transportation systems, and computer vision. From 1992 to 1993, he was a Visiting Researcher at McMaster University, Hamilton, On, Canada. His research interests are computer vision and optimization problems in the Intelligence Transport System.

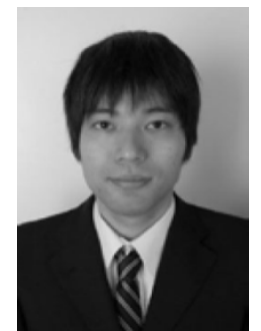

Gou Koutaki, received the B.Eng., M.Eng., and Ph.D.degree from Kumamoto University, Kumamoto, Japan, in 2002, 2004, and 2007, respectively. From 2007 to 2010, he was with Production Engineering Research Laboratory, Hitachi Ltd. He is currently at Priority Organization for Innovation and Excellence, Kumamoto University. He is engaged in research on image processing and pattern recognition of the Intelligence Transport System. 